\title{
Alternative approach to sums of dilates
}

\author{
Rafał Bystrzycki $^{1}$ and Tomasz Schoen ${ }^{2}$ \\ ${ }^{1}$ Department of Discrete Mathematics, Adam Mickiewicz University \\ ul. Umultowska 87, 61-614 Poznań, Poland \\ e-mail: rafbys @amu.edu.pl \\ ${ }^{2}$ Department of Discrete Mathematics, Adam Mickiewicz University \\ ul. Umultowska 87, 61-614 Poznań, Poland \\ e-mail: schoen@amu.edu.pl
}

Received: 2 May 2017

Accepted: 7 May 2018

\begin{abstract}
We investigate the size of the sets $\lambda_{1} \cdot A+\cdots+\lambda_{h} \cdot A$, where $\lambda_{i}$ are integers. Specifically, we look for upper bounds in terms of the doubling constant $K=\frac{|A+A|}{|A|}$. We also examine some situations in which those bounds can be significantly strengthened.
\end{abstract}

Keywords: Sumsets, Dilates, Ruzsa triangle inequality, Chang covering lemma.

2010 Mathematics Subject Classification: 11P70.

\section{Introduction and known results}

One of the classical results in additive combinatorics is Plünnecke inequality, bounding the maximal size of the set of sums of $k$ elements of $A$ by $K^{k}|A|$. Natural generalization of the problem of bounding size of the set of sums of $k$ elements is a problem of finding a good bound for the size of set of sums of the form $\lambda_{1} a_{1}+\ldots+\lambda_{k} a_{k}$ for some given integers $\lambda_{1}, \ldots, \lambda_{k}$ (in Plünnecke inequality they are all equal \pm 1 ), where $a_{1}, \ldots, a_{k}$ are elements of $A$. In this case until recently there were no known bounds out of those easily following from Plünnecke inequality. Breakthrough result was obtained in 2008 by Boris Bukh, who used binary expansion to get a bound in terms of logarithms of number $\left|\lambda_{i}\right|$ rather than those numbers themselves. He proved the following theorem. 
Theorem 1 ([1]). Let $\lambda_{1}, \ldots, \lambda_{h}$ be given integers and let $A \subset \mathbb{Z}$. If either $|A+A| \leq K|A|$ or $|A-A| \leq K|A|$, then $\left|\lambda_{1} \cdot A+\cdots+\lambda_{h} \cdot A\right| \leq K^{p}|A|$ where

$$
p=7+12 \sum_{i=1}^{h} \log _{2}\left(1+\left|\lambda_{i}\right|\right) .
$$

In particular, this result can be presented in the following simpler form:

Corollary 1. If $|A+A| \leq K|A|$ and $\left|\lambda_{i}\right| \leq 2^{r}$ then

$$
\left|\lambda_{1} \cdot A+\cdots+\lambda_{h} \cdot A\right| \leq K^{O(r h)}|A| .
$$

Bukh himself supposed that this result can be further improved in case where there are many summands involved. Slight improvement was recently obtained by Bush and Zhao, who proved the theorem below.

Theorem 2 ([2]). If $|A+A| \leq K|A|$ and $\left|\lambda_{i}\right| \leq 2^{r}$ then

$$
\left|\lambda_{1} \cdot A+\cdots+\lambda_{h} \cdot A\right| \leq K^{O\left(\frac{(r+h)^{2}}{\log (r+h)}\right)}|A|
$$

The main innovation in their proof is the use of graph theoretic methods. The main aim of this paper is to improve this bound using different (more direct) method.

It seems clear that if the set of $\lambda_{i}$ coefficients have some good additive properties it should be possible to get some better bounds. Formalizing this intuition is the main focus in the second part of the article. This line of investigation was started by the second-named author and Shkredov, who proved the following

Theorem 3 ([7]). Let $A \subset G$ be a finite set and $\lambda_{i} \in \mathbb{Z} \backslash\{0\}$. Suppose that $|A+A| \leq K|A|$, then

$$
\left|\lambda_{1} \cdot A+\cdots+\lambda_{h} \cdot A\right| \leq e^{O\left(\log ^{8} K\right)\left(h+\log \left(\sum_{i}\left|\lambda_{i}\right|\right)\right)}|A| .
$$

The novelty here is that the result shows that the problem turns out to be much easier for some specific choice of parameters $K$ and $h$, i.e. when $h$ is sufficiently large compared to $K$.

\section{Tools}

Basic tools we are going to use include primarily the so called Ruzsa calculus. It consists of inequalities bounding cardinalities of certain sumsets by expressions involving other sumsets. In our arguments we are going to use the following inequality.

Lemma 1 ([5, Sum triangle inequality]). For any finite $X, Y, Z \subset \mathbb{Z}$ we have

$$
|X+Z| \leq \frac{|X+Y||Y+Z|}{|Y|} .
$$

It is analogous to classical Ruzsa triangle inequality. 
Lemma 2 ([4]). For any finite $X, Y, Z \subset \mathbb{Z}$ we have

$$
|X-Z| \leq \frac{|X-Y||Y-Z|}{|Y|} .
$$

It should be remarked that in our approach we could use this inequality in place of sum triangle inequality. Using sums only makes the exposition a little bit clearer.

We are going to repeatedly use Plünnecke inequality as well as Bukh's theorem (Theorem 1).

Lemma 3 ([3, Plünnecke inequality]). If $|A+A| \leq K|A|$ or $|A-A| \leq K|A|$, then $|m A-n A| \leq$ $K^{m+n}|A|$ for all non-negative integers $m, n$.

In [7] the theorem of Sanders stated below is used to improve the bound when $K$ is small compared to $k$.

Lemma 4 ([6]). Suppose that $G$ is an abelian group and $A, S \subset G$ are finite non-empty sets such that $|A+S| \leq K \min \{|A|,|S|\}$. Then $(A-A)+(S-S)$ contains a proper symmetric $d(K)$-dimensional coset progression $P$ of size $\exp (-h(K))|A+S|$. Moreover, we may take $d(K)=O\left(\log ^{6} K\right)$ and $h(K)=O\left(\log ^{6} K \log \log K\right)$.

In the same paper the following corollary is proved, which we will use to continue investigation in this line of reasoning by proving Theorem 5 .

Corollary 2 ([7]). Let $A$ be a subset of abelian group $G$ such that $|A+A| \leq K|A|$. Then

$$
|k A| \leq\left(\frac{3 e k}{K}\right)^{O\left(K \log ^{8} K\right)}|A|
$$

for every $k \geq K$.

Covering lemmas turn out to be very useful in bounding sums of dilates. Bukh in his proof used the following result obtained by Ruzsa.

Lemma 5 ([5, Ruzsa covering lemma ]). For any non-empty sets $A, B$ in abelian group $G$ one can cover $B$ by $\frac{|A+B|}{|A|}$ translates of $A-A$.

We use another lemma proved by Chang to improve the bounds when then set of $\lambda$ coefficients has some additive structure.

Lemma 6 ([8, Chang covering lemma]). Suppose that $G$ is an abelian group and $A, S \subset G$ are finite sets with $|n A| \leq K^{n}|A|$ for all $n \geq 1$ and $|A+S| \leq L|S|$. Then there is a set $T$ with $|T|=O(K \log 2 K L)$ such that

$$
A \subset \operatorname{Span}(T)+S-S
$$




\section{Results}

Now we state our first theorem. This result and its proof should be compared to Theorem 2 .

Theorem 4. Let $|A+A| \leq K|A|$ and let $\left|\lambda_{i}\right|<2^{r}$ for $i=1, \ldots, h$. Then

$$
\left|\lambda_{1} \cdot A+\cdots+\lambda_{h} \cdot A\right| \leq K^{O\left(\frac{r h}{\log (h)}+h \log (h)\right)}|A| .
$$

Proof. Without loss of generality, we can assume that $h \geq 16$, since otherwise it follows from Theorem 1. If $r \leq \log h$, then again the claim follows from Theorem 1. We are going to show that it holds for every $h$ and $r$ using induction on $r$ with additional assumption that $\lambda_{1}=1$.

Let $S_{\lambda}=\lambda_{1} \cdot A+\cdots+\lambda_{h} \cdot A$. Take $d=\left\lfloor\frac{h}{\log h}\right\rfloor$. Write $\lambda_{i}$ as a sum $d \lambda_{i}^{\prime}+\alpha_{i}$ with $0 \leq \alpha_{i}<d$. Then with $\lambda^{\prime}=\left(1, \lambda_{2}^{\prime}, \ldots, \lambda_{h}^{\prime}\right)$ and $\alpha=\left(\alpha_{1}, \ldots, \alpha_{h}, d\right)$

$$
\frac{\left|S_{\lambda}(A)\right|}{|A|} \leq \frac{\left|S_{\alpha}(A)\right|}{|A|} \frac{\left|S_{\lambda^{\prime}}(A)\right|}{|A|}
$$

by Ruzsa triangle inequality with $X=\alpha_{1} \cdot A+\cdots+\alpha_{h} \cdot A, Y=d \cdot A$ and $Z=\lambda_{1}^{\prime} \cdot A+\cdots+\lambda_{h}^{\prime} \cdot A$.

We can bound the first term by collecting summands with the $\alpha_{i}$ and then repeatedly using Ruzsa triangle inequality. Writing $k_{i}$ for the number of summands with $\alpha_{j}=i$ we get

$$
\begin{aligned}
& \frac{\left|S_{\alpha}(A)\right|}{|A|}=\frac{\left|k_{1} 1 \cdot A+\cdots+k_{d-1}(d-1) \cdot A\right|}{|A|} \leq \\
& \leq \frac{\left|\left(k_{1}+1\right) A\right|}{|A|} \frac{|A+2 \cdot A|}{|A|} \prod_{i=2}^{d-1}\left(\frac{\left|\left(k_{i}+2\right) i \cdot A\right|}{|A|} \frac{|i \cdot A+(i+1) \cdot A|}{|A|}\right) \frac{|d \cdot A+d \cdot A|}{|A|}
\end{aligned}
$$

At each step we used Ruzsa triangle inequality twice: first time with $\left.X_{i}^{(1)}=k_{i} i \cdot A, Y_{i}^{(1}\right)=i \cdot A$ and $Z_{i}=k_{i+1}(i+1) \cdot A+\cdots+k_{d-1}(d-1) \cdot A$ and then the second time with $X_{i}^{(2)}=i \cdot A$, $Y_{i}^{(2)}=(i+1) \cdot A$.

Using Plünnecke inequality to bound each term with repeated summand and Theorem 1 to bound expressions with different summands we obtain

$$
\frac{\left|S_{\alpha}(A)\right|}{|A|} \leq K^{\sum_{i=1}^{d-1}\left(k_{i}+2\right)+1} K^{O(d \log d)} \leq K^{O(2 d+1+h+d \log d)}
$$

By the definition of $d$ it is

$$
\frac{\left|S_{\alpha}(A)\right|}{|A|} \leq K^{O(h)}
$$

To bound the second term we can use induction assumption. By our additional assumption $\left(\lambda_{1}=1\right)$ there will be only at most $h$ summands. By assumption on $h$ and the definition of $d$ the number of bits in $\lambda$ 's will drop by at least $\frac{1}{2} \log h$. Hence

$$
\frac{\left|S_{\lambda}(A)\right|}{|A|} \leq K^{O(h)} K^{O\left(\left(r-\frac{1}{2} \log h\right) \frac{h}{\log h}+h \log h\right)} \leq K^{O\left(\frac{r h}{\log (h)}+h \log (h)\right)}|A|
$$

Without assumption on $\lambda_{1}$ we can use the theorem for the set $A+\lambda_{1} \cdot A+\cdots+\lambda_{h} \cdot A$ with $(h+1)$ summands, which contains a translate of the original set. It follows that in this case the claim holds with slightly larger constant implicit in $O()$ notation. 
Our next theorem applies to the case when $K$ is much smaller than $h$. It shows that then the dependence on $h$ becomes polynomial under those assumptions. Hence it improves on Theorem 3 in such circumstances.

Theorem 5. Let $|A+A| \leq K|A|$ and let $\left|\lambda_{i}\right|<2^{r}$ for $i=1, \ldots, h$. If $h \geq K$, then

$$
\left|\lambda_{1} \cdot A+\cdots+\lambda_{h} \cdot A\right| \leq\left(C(K) h^{f(K)}\right)^{r}|A|,
$$

where $C(K)=\frac{15 e}{2 K}$ and $f(K)=O\left(K \log ^{8} K\right)$

Proof. Again we are going to use induction on $r$ with additional assumption that $\lambda_{1}=1$. For $r=$ 1 it follows from corollary 2. To show it for greater $r$ we use binary expansion, namely we write $\lambda_{i}$ as a sum $2 \lambda_{i}^{\prime}+\alpha_{i}$ with $\alpha_{i} \in\{0,1\}$. Then with $\lambda^{\prime}=\left(1, \lambda_{2}^{\prime}, \ldots, \lambda_{h}^{\prime}\right)$ and $\alpha=\left(\alpha_{1}, \ldots, \alpha_{h}, 2\right)$ we have (just as in the proof of the previous theorem)

$$
\frac{\left|S_{\lambda}(A)\right|}{|A|} \leq \frac{\left|S_{\alpha}(A)\right|}{|A|} \frac{\left|S_{\lambda^{\prime}}(A)\right|}{|A|}
$$

We can bound the first term using corollary 2 and the fact that $2 \cdot A \subset A+A$ by

$$
\frac{\left|S_{\alpha}(A)\right|}{|A|} \leq \frac{|(h+2) A|}{|A|} \leq\left(\frac{3 e(h+2)}{K}\right)^{O\left(K \log ^{8} K\right)} .
$$

Using induction assumption to bound the second term we get

$$
\frac{\left|S_{\lambda^{\prime}}(A)\right|}{|A|} \leq\left(C(K) h^{f(K)}\right)^{r-1}
$$

Multiplying the last two equations we get

$$
\frac{\left|S_{\lambda}(A)\right|}{|A|} \leq\left(\frac{3 e(h+2)}{K}\right)^{O\left(K \log ^{8} K\right)}\left(C(K) h^{f(K)}\right)^{r-1}=\left(C(K) h^{f(K)}\right)^{r} .
$$

It finishes the proof of the claim with additional assumption. We get rid of this assumption in the same way as in the previous proof.

Our last theorem considers the case when $\Lambda$ - the set of $\lambda_{i}$ coefficients - has some additive structure. In such setting spectacular improvement is possible.

Theorem 6. Let $|A+A| \leq K|A|$ and let $\Lambda \subset\left[2^{r}\right]$. Furthermore, assume that $|\Lambda+\Lambda|<L|\Lambda|$. Then

$$
\left|\lambda_{1} \cdot A+\cdots+\lambda_{h} \cdot A\right| \leq K^{O((h+r) L \log L)}|A|
$$

Proof. By Chang covering lemmma (with $A=\Lambda, K=L, S=\{0\}$ ), we know that there is a set $\Gamma$ such that $\Lambda \subset \operatorname{Span}(\Gamma)$ and $|\Lambda|=O(L \log 2 L)$. Write each $\lambda_{i}$ as a sum $\lambda_{i}=\sum_{j=1}^{|\Gamma|} \epsilon_{i, j} \gamma_{j}$. Then

$$
\left|\lambda_{1} \cdot A+\cdots+\lambda_{h} \cdot A\right|=\left|\sum_{i=1}^{h}\left(\sum_{j=1}^{|\Gamma|} \epsilon_{i, j} \gamma_{j}\right) \cdot A\right| \leq\left|\sum_{i=1}^{h} \sum_{j=1}^{|\Gamma|} \epsilon_{i, j} \gamma_{j} \cdot A\right|=\left|\sum_{j=1}^{|\Gamma|} \sum_{i=1}^{h} \epsilon_{i, j} \gamma_{j} \cdot A\right|
$$


We can use Ruzsa triangle inequality twice (the first time with $X_{1}=\sum_{i=1}^{h} \epsilon_{i, 1} \gamma_{1} \cdot A, Y_{1}=$ $\gamma_{1} \cdot A, Z=\gamma_{1} \cdot A \sum_{i=2}^{h} \epsilon_{i, j} \gamma_{j} \cdot A$, the second time with $X_{2}=\gamma_{1} \cdot A$ and $Y_{2}=\gamma_{2} \cdot A$ ) to bound the last expression by

$$
\left|\sum_{j=1}^{|\Gamma|} \sum_{i=1}^{h} \epsilon_{i, j} \gamma_{j} \cdot A\right| \leq \frac{\left|\sum_{i=1}^{h} \epsilon_{i, 1} \gamma_{1} \cdot A+\gamma_{1} \cdot A\right|}{|A|} \frac{\left|\gamma_{1} \cdot A+\gamma_{2} \cdot A\right|}{|A|}\left|\gamma_{2} \cdot A+\sum_{j=2}^{|\Gamma|} \sum_{i=1}^{h} \epsilon_{i, j} \gamma_{j} \cdot A\right| .
$$

Using Plünnecke inequality to bound the term with repeated (up to sign) summand and Theorem 1 to bound the expression with different summands we obtain

$$
\left|\sum_{j=1}^{|\Gamma|} \sum_{i=1}^{h} \epsilon_{i, j} \gamma_{j} \cdot A\right| \leq K^{O((h+1)+r)}\left|\gamma_{2} \cdot A+\sum_{j=2}^{|\Gamma|} \sum_{i=1}^{h} \epsilon_{i, j} \gamma_{j} \cdot A\right|
$$

Continuing in this way, we can prove by induction that

$$
\left|\sum_{j=1}^{|\Gamma|} \sum_{i=1}^{h} \epsilon_{i, j} \gamma_{j} \cdot A\right| \leq K^{O((h+2)+r)}\left|\gamma_{k} \cdot A+\sum_{j=k}^{|\Gamma|} \sum_{i=1}^{h} \epsilon_{i, j} \gamma_{j} \cdot A\right|
$$

which for $k=|\Gamma|$ gives the claim (after another application of Plünnecke inequality).

We conclude with a simple lemma showing once again how additive structure of $\Lambda$ may influence the bounds.

Lemma 7. For any $i, j$, if we take $\lambda_{i}^{\prime}=\lambda_{i} \pm \lambda_{j}$ and $\lambda_{k}^{\prime}=\lambda_{k}$ for $k \neq i$, we have

$$
\frac{\left|S_{\lambda}(A)\right|}{|A|} \leq K^{3} \frac{\left|S_{\lambda^{\prime}}(A)\right|}{|A|}
$$

Proof. To see this we use the fact that $S_{\lambda}(A) \subset S_{\lambda^{\prime}} \mp \lambda_{j} \cdot A$. Let $\lambda_{j}^{\prime \prime}=0$ and $\lambda_{k}^{\prime \prime}=\lambda_{k}^{\prime}$ for $k \neq j$. Now we use Ruzsa triangle inequality with $X=S_{\lambda^{\prime \prime}}, Y=\lambda_{j} \cdot A$ and $Z=\lambda_{j} \cdot A \mp \lambda_{j} \cdot A$.

$$
\frac{\left|S_{\lambda}(A)\right|}{|A|} \leq \frac{\left|S_{\lambda^{\prime}}(A) \mp \lambda_{j} \cdot A\right|}{|A|} \leq \frac{\left|S_{\lambda^{\prime}}(A)\right|}{|A|} \frac{\left|\lambda_{j} \cdot A+\lambda_{j} \cdot A \mp \lambda_{j} \cdot A\right|}{\left|\lambda_{j} \cdot A\right|} .
$$

Now (1) follows from Plünnecke inequality.

\section{References}

[1] Bukh, B. (2008) Sums of dilates, Combin. Probab. Comput., 17, 627-639.

[2] Bush, A. \& Zhao, Y. (2017) New upper bound for sums of dilates, Electon. J. Comb., 24 (3), \# P3.37.

[3] Plünnecke, H. (1970) Eine zahlentheorische anwendung der graphtheorie, J. Reine Angew. Math., 243, 171-183. 
[4] Ruzsa, I. (1996) Sums of finite sets. In Number Theory: New York Seminar, 281-293.

[5] Ruzsa, I. (2009) Sumsets and structure. In Combinatorial Number Theory and Additive Group Theory, 87-210.

[6] Sanders, T. (2012) On the Bogolyubov-Ruzsa Lemma, Anal. PDE, 5, 627-655.

[7] Schoen, T. \& Shkredov I. D. (2016) Additive dimension and a theorem of Sanders, J. Aust. Math. Soc., 100, 124-144.

[8] Tao, T.C. \& Vu, H.V. (2006) Additive combinatorics, volume 105 of Cambridge Studies in Advanced Mathematics, Cambridge University Press, Cambridge. 